\title{
HYBRID SIMULATION SYSTEM FOR ANALYSIS OF THE SPECIALIZED MOBILE SYSTEMS
}

\author{
A. Maslowski, A. Andrzejuk, A. Czerniewska - Majewska, P. Szynkarczyk
}

Robotized Mobile Systems Division (ZUM)

Research Institute for Automation and Measurements (PIAP)

A1. Jerozolimskie 202, 02-486 Warsaw, POLAND

\begin{abstract}
The aim of this paper is to present the simulation system for analysis of the specialized mobile systems, which are equipped with a human operator - supported computer system (on board or tele-operator). Simulation is performed in real time, using a computer system and a mobile robot. Visualization of the simulation of the environment, movements of the mobile base and behaviour of the human operator is done by a computer animation and partly by a mobile robot and its physical environment. Correct behaviour of the control unit in different environmental conditions including a detection of obstacles using different sensors could be observed using mobile robot moving in simulated environment and configured especially to experimental requirements.
\end{abstract}

\section{INTRODUCTION}

Specialized mobile systems are dedicated to be used in specific environments, always in hazardous conditions when replacement or assistance to the human operator are required, in his activities. Main part of such system are computer controlled actuators according to a specified algorithm. Actuators are performing physical part of the robot's task using its manipulators. Such robots are equipped with sensors for collecting information about the environment and parameters describing the state of the particular parts of the robot.

Recognize and Control Devices (RCD) are main part of the mobile system which is used for research in the ZUM-PIAP laboratory. Proper work of the RCD is important for the mobile system to perform any task precisely (e.g. assisting human or autonomous activities).

Great Number of probable problems concerning work of the specialized mobile systems causes requirements for complex research into the construction of the sensors, actuators and control algorithms. As an answer to such requirements, simulation project of the specialized systems was developed in ZUM-PIAP laboratory. Simulation research is possible without risk of damaging real devices in case of control failure and with the possibility of spending less money. In addition, research is possible on based on such assumptions which allow reconstructions during development of given devices or units of specialized mobile systems. 


\section{SIMULATION SYSTEM}

Simulation system designed in the Robotized Mobile Systems Division at the Research Institute for Automation and Measurements consists of the following subsystems [11], [3]:

a) simulation of the Recognize and the Control Devices (RCD),

b) simulation of the specialized mobile system,

c) simulation of the environment,

d) simulation of the human operator,

e) simulation of the intermediary device (man-machine interface),

f) simulation of the actuators,

g) simulation of the sensors.

Block diagram of the simulation system is shown below in Fig. 1 .

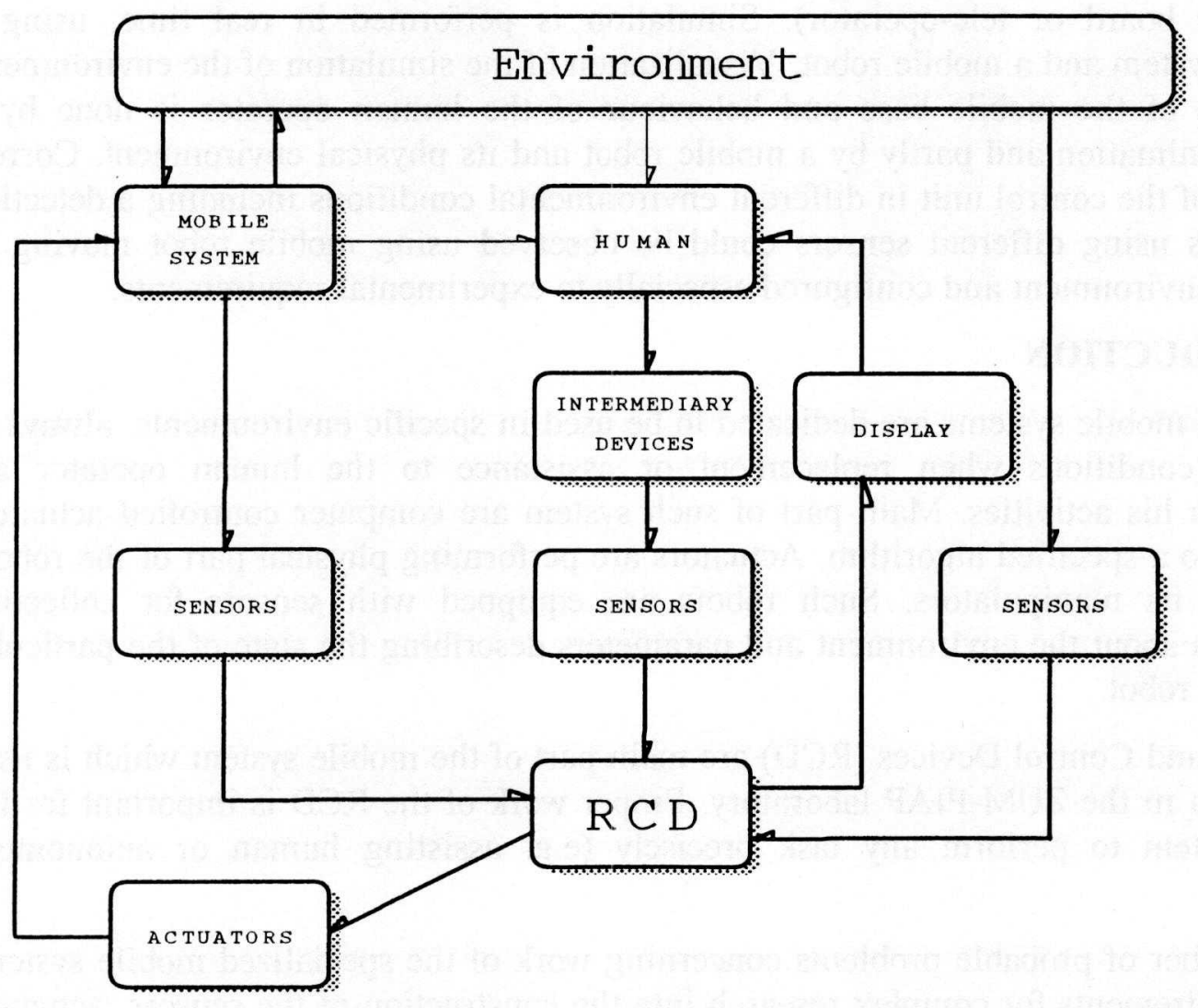

Fig. 1. Block diagram of the simulation system. 


\subsection{Recognize and Control Devices (RCD)}

RCD consists of two main parts (Fig 2.). The 'Recognize Device' is the first element of the system, which collects sensoric data from the environment, from the interior of the mobile base and finally from a human operator. Synthetic description of the whole situation (interior state of the mobile base, environment state and human operator wishes) is the result of the work of that unit. Those information can be also displayed for the operator. Actual requirements of the operator and data from the recognized situation are transmitted to the second, control unit. The 'Control Device' based on the delivered information generates commands for the actuators.

Environment is recognized mainly by a real time vision system. After all necessary processing, obtained data is sent to control device and to the human driver to improve sensoric abilities of the man-machine system. Vision system is monitoring the environment in front of the mobile system, recognizing his path, detecting and recognizing moving or static objects.

Localization of the other moving objects and obstacles on both sides and behind the mobile system is observed with the use of ultrasonic, infra-red and laser sensors. Measurements of distances and velocities of recognized and pointed by a vision system obstacles, is done with the use of those sensors.

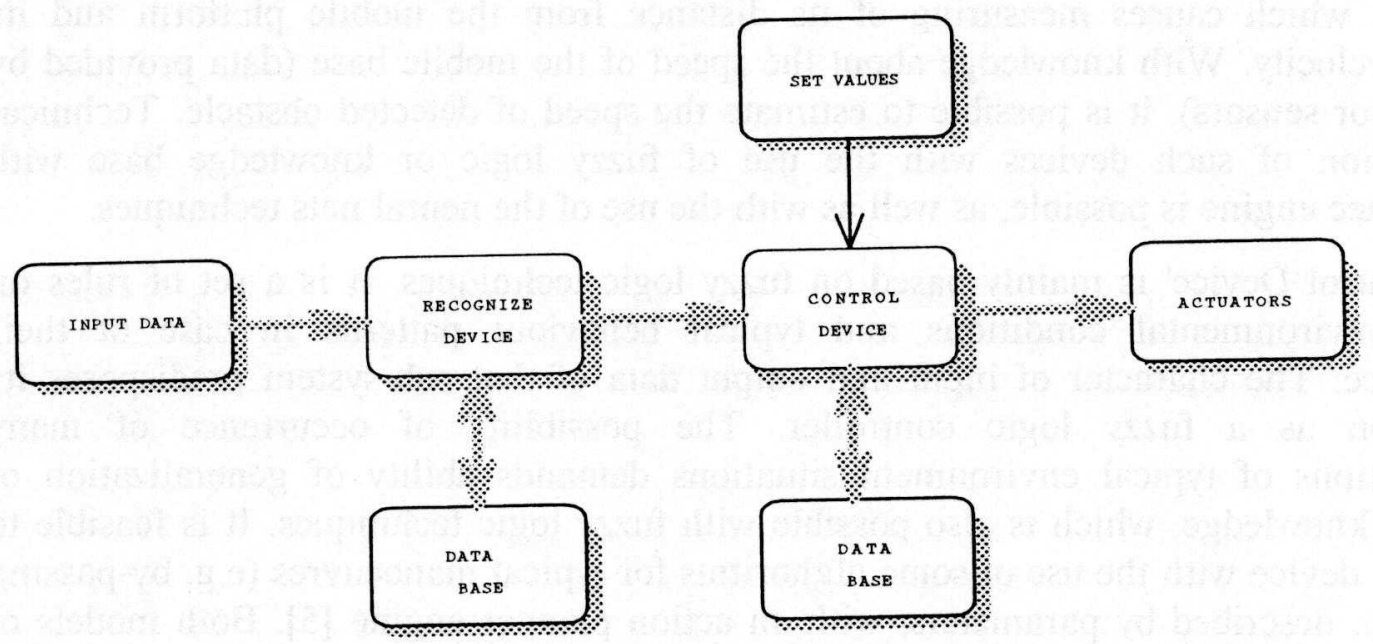

Fig. 2. Block diagram of the RCD

Description of the environment, interior state of the mobile base and human requirements are the input to the 'Control Device'. This data is provided in a synthetic short form. The aim of the 'Control Device' is to make decisions and execute proper motions of the mobile platform, using data provided by the recognized device in accordance with the environmental conditions and operator's requirements and intentions.

The 'Recognize Device' and the 'Control Device' must have the ability of using some knowledge to complete their tasks. In case of 'Recognize Device' the knowledge base must assume: elementary knowledge of the environment, knowledge of method of transferring data provided by sensors, into suitable description for control unit and for human operator (by intermediary devices). In case of 'Control Device', the knowledge 
must assume elementary knowledge of: mobile system's environment, actuators' dynamics, mobile platform's dynamics, behaviour of the human operator and methods and ways of correct behaviour in different environment situations.

RCD must work in real time (maximum speed of mobile base with computer assisted operator depends on their speed of calculations) and must be reliable. Because of complicated tasks to perform, demanding level of operated knowledge, variety of possible environmental conditions (control device) and possibility of measurement interferences (recognize device), construction of both units assume artificial intelligence problems. The use of the neural networks and fuzzy logic methods has been tested in this research.

The 'Recognize Device' was developed with the use of neural networks mainly. Vision data after pre-processing (filtering, edge extraction and other basic operations) - is transferred to neural net. At the moment, research on adapting back propagation multilayer net and fuzzy ART map net is in progress. The main task of the net is creating the image data processing to detect obstacles on mobile platform's path and to estimate the mobile platform's position. Information of position of the detected obstacles is used to correlate them with other sensors' distance measurements. In that way in 'recognize device', process of linking vision data with measurements of range finders is performed. For example after processing the information such as "obstacle in front on a path" is obtained, which causes measuring of its distance from the mobile platform and its relative velocity. With knowledge about the speed of the mobile base (data provided by the interior sensors), it is possible to estimate the speed of detected obstacle. Technical construction of such devices with the use of fuzzy logic or knowledge base with intelligence engine is possible, as well as with the use of the neural nets techniques.

The 'Control Device' is mainly based on fuzzy logic techniques. It is a set of rules on typical environmental conditions and typical behaviour patterns in case of their occurrence. The character of input and output data of that sub-system predisposes its realization as a fuzzy logic controller. The possibility of occurrence of many modifications of typical environment situations demands ability of generalization of operated knowledge, which is also possible with fuzzy logic techniques. It is feasible to built that device with the use of some alghoritms for typical manoeuvres (e.g. by-passing obstacles), described by parameters, with an action planner engine [5]. Both models of control device have been tested in project by simulated experiments.

\subsection{Environment Simulation}

Providing data of actual environmental conditions and other environmental parameters are essential for proper work of the whole simulation system. This is performed by the environment simulator sub-system. Full description of positions, shapes and velocities of the obstacles is required, as well as a description of static objects. The state of the environment is described by parameters and their random composition or a result of the actions of the operator of the simulation system. Other task of the environmental simulator is to visualize the progress of simulation (computer animation of all parameters, top down view and the operator's of the mobile platform view). Simulation of the environment can be performed in two ways. Full computer simulation is the first one. In that case whole environment and all of its parameters are described in form of matrices (their changes are computed by a simulation program). The second method is to 
simulate mobile system's environment by an experimental laboratory environment model.

\subsection{Simulation of the Human Operator}

The human operator could be represented by a computer simulation or could be a real human controlling a mobile platform from the inside of the simulator's cabin, where real cockpit of the mobile system is mounted. Such simulator hardware is going to be designed and built in this research project.

One of the possible options for the computer simulation is the human operator model, based on a neural net learning from human behaviour while the test operator of the system drives a simulated mobile base, and then acting during tests as the human would do. Research on use of the back propagation multi-layer net is ongoing.

Research using simulation system demands many experiments with presence of the human operator. In that case real human in a simulator's cabin can be replaced with a human-like model simulating human's behaviour. It is useful also in case of simulating different human operator's errors. When simulation is performed not in real time (it is possible to do so in this simulation system), model of the human behaviour is particularly indispensable.

\subsection{Simulation of the Sensors}

The sensors sub-system is divided into exterior sensors, interior sensors and sensors mounted in the intermediary devices. Exterior sensors are the sensors providing data concerning the mobile system environment. Those are the following: vision system (providing image of the environment in front of the robot), infra-red, ultrasonic and laser sensors (providing distances to the obstacles around the mobile system) and additional sensors (providing data concerning surface state and other external conditions). Interior sensors are there to measure speed, acceleration, rotational speed of the engine, brake force, steering angle, power consumption etc.

Sensors are supposed to be simulated with the use of simulation of the physical phenomenons concurring work of the sensors [12]. Another way of simulation of the exterior sensors is the use of the mobile robot equipped with laser, ultrasonic, tactile and infra-red sensors. Data obtained from experiments with mobile robot in several environmental situations will allow to design proper locations of the sensors on real mobile system and their types and parameters. Analysis of measurements obtained from experiments with robot will also allow to answer the question: 'What are the main problems to solve in the real vehicle moving in a the real environment?' During computer simulation of the sensors, errors could only be estimated.

\section{RESEARCH}

Two modes of operation of the mobile system are planned to be researched in this project ([3], [5]). The first one is named 'Safety Monitoring and Warning'. Human operator is driving a mobile platform according to his abilities, RCD is only watching his actions and environmental changes, informing him about safety margin and any additional data (e.g. positions and velocities of other moving obstacles around mobile platform). RCD forces its decisions (or warns human operator in a simpler to construct version) only in case of decreasing safety coefficient below assumed margin, when human makes 
mistakes, loses attention or is unable to perform necessary fast actions. The second mode of operation of RCD is 'drive-by-wire'. In that case man-machine interface (intermediary devices) senses human operator's intentions. In addition human is relieved from a low level operations (e.g. compensory steering control). RCD input is data on environmental and interior state of mobile system (provided by sensors), human intentions (intermediary devices), and set values such as human decisions (e.g. according to style of driving - fast or slow). Elements of autonomy are planned in this mode. Human would be able to order performing one of autonomous functions (e.g. obstacle by-passing).

Research can be performed in two modes of operation of the simulation system: with the use of the vehicle and environmental models, with the use of the mobile robot and his physical environment.

The aim of the project is to build a real time simulation system with on-line computer graphic animation. Such task can be performed only by a very fast computer equipped with very fast processor and other input/output devices. The other solution of the problem would be to use a real time multitasking operating system working in the net of computers. QNX 4.2 operating system [13] with net of IBM PC can manage those requirements.

As a specific research tool, mobile robot NOMAD 200 (made by NOMADIC TECHNOLOGIES, INC, U.S.A.) [12] is used. It is three degrees of freedom (DOF) mobile robot (translation, steering and rotation of the turret). On-board computer system consists of master $486 / 66 \mathrm{MHz}$ IBM PC communicating with slave processors through shared memory. Slave processors are the following: Sensor interface processor (MC68HCllF1), Motion Control processor (68008) and Bus Peripherals, Hard Disk and Vision System hardware. Robot control can be performed manually by joystick or automatically by a program in C or LISP, both from the on-board system or from the Host Computer System by radio modem (range of modem: $200 \mathrm{~m}$ ). The Robot is equipped with the following sensor systems: Tactile System, Infrared Ranging System, Sonar Ranging System, Laser Ranging System. Robot is provided with software including $\mathrm{C}$ libraries and simulator of the environment, sensors and robot's motions. Real Time Vision System DT2867 is also installed on robot. Robot as a part of the simulation system is connected to a computer QNX network with use of the radio modem (Fig. 3.)

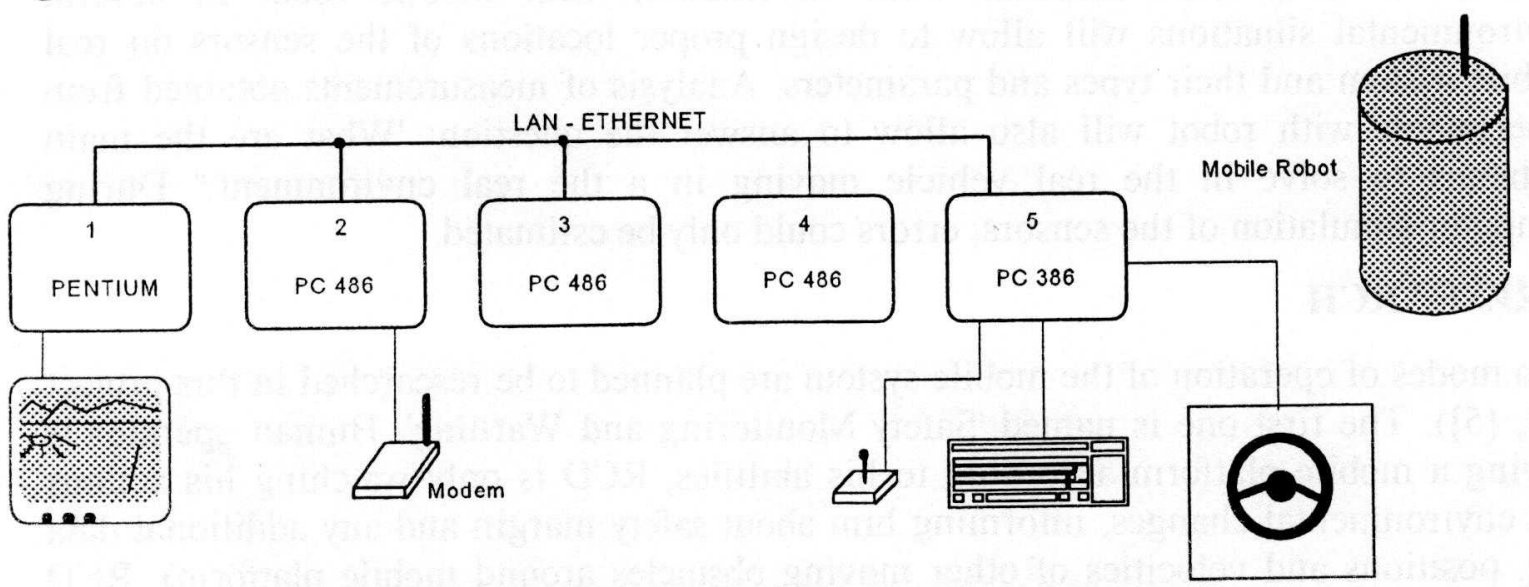

Fig. 3. Hybrid Simulation System with mobile robot 


\section{CONCLUSIONS}

Described simulation system is a very flexible system which can be used for simulation of different models of moving platforms, sensors, actuators and other modules for versatile research in specialized mobile systems. All system research can be preceded by simulation of the sub-systems. Combining full computer simulation with experiments using the real mobile robot, ensures taking into account all possible circumstances which can occur in the use of the real mobile specialized system.

Two basic configurations of the simulation system for human operator's computer assistance are designed, to subsidize computer simulation with graphics animated visualization and hybrid simulation with a mobile robot. These configurations should allow the design of many specialized mobile systems at a low cost and without risk of collisions at high speed of the real mobile platform, which could be built in the next stage, after all the neccesary experiments with the mobile robot.

Simulation system is working with the use of the original PIAP-ZUM software, the operating scheme ONX 4.2 and the NOMAND 200 mobile robot software.

\section{References}

1. A. Maslowski, A. Andrzejuk, A. Czerniewska - Majewska, P. Szynkarczyk, "Structure of the Hybrid System for the Robotized Vehicles Simulation", Research Raport PIAP No 7020, December 1993, (in Polish).

2. R. Navon, "Determination of Optimal Construction Robot Configuration", Israel Journal of Technology Vol. 251989 pp. 71-76.

3. A. Maslowski, P. Szynkarczyk, A. Czerniewska - Majewska, A. Andrzejuk, " Hybrid Computer - Experimental Simulation System for Research of the Robotized Vehicles", proceedings of 27th ISATA, 1994, Aachen, Germany.

4. R. Navon and A. Warszawski, "Factors Affecting the Productivity of a Multi-Purpose Interior Robot", The International Journal of Construction Information Technology, Vol. 1 No.1, pp. 59-78.

5. Satonobu J., Kojima K., Takahashi H., Kutami A., "The Development of a Computer System for Autonomous Vehicle Control", MAZDA Motor Corp., Technical Research Center (Yokohama), SAE 923036.

6. R. Navon, "Cost and weight analysis of a construction robot at the conceptual design stage", Manufacturing Review vol 3, no 4, December 1990.

7. P. Reignier, "Fuzzy logic techniques for mobile robot obstacle aviodance", proceedings of the International Workshop on Intelligent Robotics Systems '93, Zakopane, Poland, July 20-24, 1993.

8. Hock C., Dickmanns E.D., "Intelligent Navigation for Autonomous Robots Using Dynamic Vision", XVII Congress of the Intern. Society for Photogrammetry and Remote Sensing (ISPRS), Washington, D.C. 1992.

9. M. Devy, P. Fillareau, Marie-Christine Tatard, "Localization of an Autonomous Mobile Robot from 3D Depth Images in a Structured Environment", EUREKA EU-18 project

10. E. D. Dickmanns, " Expectation based dynamic scene understanding", Universität der Bundeswehr München / LRT/ WE 13 / IB / 92-2. 
11. D. A. Pomerleau "Knowledge-based Training of Artificial Neural Networks for Autonomous Robot Driving" (author's information).

12. "Nomad 200 User's Guide", Rev. Date 5/93, "The Sensus 500 User's Guide", Rev. Date 5/93, "Nomadic Host Software Development Environment (Release 2.1)", Rev. Date 11/93, documentation of the Nomad 200 mobile robot manufactured by Nomadic Technologies Inc. U.S.A.

13. "QNX 4 Operating System, System Architecture", Quantum Software Sysyems Ltd, July 1993.

14. R. P. Lippmann, "An Introduction to Computing with Neural Nets", IEEE ASSP Magazine, April 1987. 